\title{
Is repeated retreatment necessary for HIV-negative serofast early syphilis patients?
}

\author{
YONG LIU ${ }^{1-4}$, QUEQIAO BIAN ${ }^{1-4}$, SHUHUAN ZHANG ${ }^{1-4}$, JUN WANG ${ }^{1-4}$, ZHENMING WANG $^{2-5}$ and JUNYUE LI ${ }^{2-5}$ \\ ${ }^{1}$ Department of Dermatology and Sexually Transmitted Diseases, The Third Central Hospital of Tianjin; \\ ${ }^{2}$ Tianjin Key Laboratory of Artificial Cells; ${ }^{3}$ Artificial Cell Engineering Technology and Research Center of \\ The Public Health Ministry; ${ }^{4}$ Tianjin Institute of Hepatobiliary Disease; ${ }^{5}$ Clinical Laboratory Department, \\ The Third Central Hospital of Tianjin, Tianjin 300170, P.R. China
}

Received May 28, 2019; Accepted October 11, 2019

DOI: $10.3892 /$ etm.2019.8180

\begin{abstract}
A persistent non-treponemal serological response can be observed in patients with syphilis after treatment and is referred to as serofast. This status makes it difficult for clinicians to judge the curative effect of treatment, particularly in patients with early syphilis. In the present study, a total of 114 eligible serofast patients treated between January 2009 and June 2016 were retrospectively analyzed. All patients were subjected to rapid plasma reagin (RPR) serological tests and followed up for 24 months. The patients who remained serofast after initial therapy were given the first retreatment, and at 12 months, those who were still serofast received a second retreatment. After the first retreatment (6 months), $33.3 \%$ of the subjects $(38 / 114)$ were serologically cured ( $\geq 4$-fold decline in RPR titer). At 24 months, the patients that had achieved serological cure accounted for $23.7 \%(18 / 76)$ of the patients that received the second retreatment. Furthermore, $26.3 \%$ of subjects that achieved serological cure (10/38) and had not been further treated after the first retreatment spontaneously presented with $a \geq 4$-fold decline in RPR titer or negative status. In conclusion, the present study indicated that in patients with early syphilis and serofast status after initial treatment, retreatments may not provide any significant benefit. The second retreatment did not significantly improve the patient's serological cure rate. There is no evidence that patients with early syphilis and serofast should receive multiple retreatments, in spite of this being commonly performed in clinical practice.
\end{abstract}

Correspondence to: Mr. Yong Liu, Department of Dermatology and Sexually Transmitted Diseases, The Third Central Hospital of Tianjin, 83 Jintang Road, Hedong, Tianjin 300170, P.R. China E-mail: liuyongtj@163.com

Key words: syphilis, serofast, benzathine penicillin, retreatment, serological response

\section{Introduction}

Caused by Treponema pallidum (TP), syphilis is a chronic and infectious disease that may invade multiple organs (1). At present, the prevention and cure of syphilis remain a great challenge. In spite of certain breakthroughs regarding the laboratory cultivation of TP, detection of its DNA and specific proteins remains difficult in clinical practice (2). Whether syphilis is curable or not depends on the rapid plasma reagin (RPR) antibody test. Serological cure is defined as either a negative RPR or $\geq 4$-fold decrease (2-fold dilution) in titer at 6 months following therapy (3). Serofast is defined as either no change in RPR titer or an up to 2-fold decrease (1-fold dilution) or increase in titer following initial therapy or retreatment $(4,5)$. Retreatment for syphilis is recommended by the syphilis treatment guidelines of the USA, UK and China when additional follow-up cannot be ensured (6-8). However, the number of retreatments and the treatments' effectiveness remain controversial. A relatively small number of studies have been performed to examine the frequency of retreatments in early syphilis and serofast patients. Seña et al (5) and Wang et al (9) re-treated early syphilis participants who were serofast at 6 months and analyzed the serological response after retreatment and an additional 6 months of follow-up. These two studies demonstrated a moderately incremental benefit of retreatment in serofast early syphilis patients. It remains unclear whether serofast patients require further retreatment after the first retreatment.

Over the last 20 years, the incidence of syphilis has been rapidly increasing in China (10). Syphilis has become one of the three major infectious diseases in China, which raises the concern of clinicians. Serofast is frequently observed in patients with early syphilis (primary, secondary or latent) after treatment (11). This status makes it difficult for clinicians to judge the curative effects of treatment. Due to the associated anxiety of affected patients, penicillin is frequently overused by clinicians in certain hospitals (7). Serofast patients with syphilis receive penicillin treatments every 3-6 months over 2-3 years. In this scenario, the treatment of syphilis and the prognosis of patients require further investigation. In the present study, data of patients with early syphilis and serofast status after the first and second retreatment were analyzed in order to contribute to the determination of the optimal therapeutic schedule. 


\section{Materials and methods}

Subjects. The present retrospective study considered 1,036 consecutive patients with early syphilis (primary, secondary or early latent) treated as outpatients at Tianjin Third Central Hospital (Tianjin, China) between January 2009 and June 2016. A flow chart of the study design is presented in Fig. 1. A total of 114 eligible serofast patients were included in the present study. All clinical data, including medical history, process of treatment and results of laboratory tests recorded by clinicians of the outpatient department were analyzed. According to the diagnostic criteria of the China Centers for Disease Control and Prevention (8), early syphilis consists of primary syphilis, secondary syphilis and early latent syphilis. Primary syphilis is characterized by more than one chancre and inguinal lymphadenopathy, as well as laboratory confirmation of TP in clinical specimens by RPR and particle agglutination assay for antibody to TP (TPPA), or TP haemagglutination. Secondary syphilis is characterized by skin rash and lymphadenopathy, and confirmed by laboratory testing results for syphilis (12). Early latent syphilis is defined as patients within a year of asymptomatic syphilitic infection supported by positive laboratory testing results and normal cerebrospinal fluid (CSF).

All patients with early syphilis have positive results of serologic examinations (RPR and TPPA). The RPR antibody test was used to evaluate the curative effect of treatment in the present study. Serological cure was defined as either a negative RPR or a $\geq 4$-fold decrease (2-fold dilution) in titer at 6 months following therapy (3). Serofast is defined as either no change in RPR titer or a 2 -fold decrease (1-fold dilution) or increase in titer following initial therapy or retreatment.

The inclusion criterion was serofast early syphilis, while the exclusion criteria were as follows: i) Tertiary syphilis; ii) HIV-positive status; iii) CSF abnormality; iv) presence of other infectious diseases or autoimmune diseases; v) drug abuse; vi) loss to follow-up. The patients were stratified into different groups based on retreatment: The 1st retreatment group comprised patients with early syphilis with a $<4$-fold decrease (2-fold dilution) in titer at 6 months following initial therapy; the 2nd retreatment group included serofast patients after the 1st retreatment; the control group for the 2nd retreatment group were patients that had achieved serological cure by 6 months following the initial therapy.

Treatment. According to the Chinese national guidelines for the treatment of syphilis, patients with no penicillin allergy received initial treatment with benzathine penicillin (administered as three doses of 2.4 million units intramuscularly each at 1-week intervals; 7.2 million units in total). Alternatives to this treatment of syphilis were doxycycline $100 \mathrm{mg}$ orally twice daily for 15 days or procaine penicillin 0.8 million units intramuscularly daily for 15 days. After the treatment, the serum RPR titers and clinical symptoms of all patients were reviewed every 3 months. At baseline (T0) and 6 months after the initial treatment (T1), the RPR test was performed on patients to evaluate the curative effect of treatment. Serofast patients were included in the 1st retreatment group. The RPR test was used to evaluate the curative effects of treatment at 12 months after initial treatment (T2). Patients who remained serofast were included in the 2nd retreatment group. At 24 months after the initial treatment (T3), the RPR test was used again to evaluate the curative effects.

Statistical analysis. All data were analyzed using SPSS (version 24.0; IBM Corp.). Prior to analysis, normality of distribution was evaluated using the Kolmogorov-Smirnov test. Categorical variables were represented as a percentage and differences between groups were assessed using the Chi-square test. Continuous variables were expressed as the median and interquartile range and were compared using the Mann-Whitney $\mathrm{U}$ test. All hypothesis testing was two-sided and $\mathrm{P}<0.05$ was considered to indicate a statistically significant difference.

\section{Results}

General information. As presented in Fig. 1, a total of 192 patients $(18.5 \%)$ were serofast at 6 months after the initial treatment and 114 eligible serofast patients were included in the present study. A total of 92 serofast patients were treated with benzathine penicillin and the remaining 22 patients received an alternative therapy. There were no significant differences in efficacy between the different treatments. The median age of the patients was 36 years (range, 36-49) and the majority were female (60.5\%). Primary syphilis accounted for $8.8 \%$ (10/114), secondary syphilis accounted for $14.0 \%$ (16/114) and early latent syphilis accounted for $77.1 \%$ (88/114; Table I).

Serological outcomes of serofast patients with early syphilis after the first retreatment. As presented in Table I, compared with serofast patients, those that achieved serological cure exhibited no significant difference in sex, age and initial treatments. However, there were more primary and secondary cases of syphilis in patients that achieved serological cure than in serofast patients ( 13.1 vs. $6.6 \%, 23.7$ vs. $9.2 \%, \mathrm{P}<0.05)$. Patients with latent syphilis more frequently achieved serofast than serological cure $(84.2$ vs. $63.2 \%, \mathrm{P}<0.05)$. After the first retreatment, $17.5 \%(20 / 114)$ of subjects exhibited a $\geq 4$-fold decline in the RPR titers compared with the initial treatment. However, when the serological response was determined in comparison with the baseline titers, it was indicated that $33.3 \%$ of patients (38/114) had achieved a serological cure. Significant differences in RPR titer at T0 (prior to initial treatment), T1 (at 6 months) and T2 (at 12 months) were observed between serofast patients and those with serological cure $(\mathrm{P}<0.05)$.

Serological outcomes in serofast patients with early syphilis after the second retreatment. The 76/114 patients (66.7\%) who remained serofast after the first retreatment received a second retreatment (Table II). After the second retreatment, a total of 18 (23.7\%) patients had a $\geq 4$-fold decline in RPR titer from their T2 (at 12 months) RPR titer. The spontaneous RPR titer change of the 38 patients serologically cured after the first retreatment were also followed up at 24 months. Of these patients, $26.3 \%(10 / 38)$ exhibited a $\geq 4$-fold decline in RPR titer or negative status compared to the T2 (at 12 months) RPR titer. There was no significant difference in RPR titer at $\mathrm{T} 3$ (at 24 months) between the first retreatment and the second retreatment group (Table III).

After two retreatments, a total of $56(49.12 \%)$ patients achieved serological cure and the remaining 58 (50.88\%) 
Table I. Characteristics and serological outcomes of serofast patients with early syphilis after the first retreatment.

\section{A, Sex}

\begin{tabular}{|c|c|c|c|c|}
\hline & Overall $(n=114)$ & Serological cure $(n=38)$ & Serofast $(n=76)$ & P-value \\
\hline Female & $69(60.5)$ & $20(52.6)$ & $49(64.5)$ & 0.34 \\
\hline \multicolumn{5}{|l|}{ B, Age } \\
\hline & Overall $(n=114)$ & Serological cure $(n=38)$ & Serofast $(n=76)$ & P-value \\
\hline Median age, years (IQR) & $36(23)$ & $33(21)$ & $36(24)$ & 0.24 \\
\hline \multicolumn{5}{|l|}{$\mathrm{C}$, Syphilis type } \\
\hline Syphilis type & Overall $(n=114)$ & Serological cure $(n=38)$ & Serofast $(n=76)$ & P-value \\
\hline Primary & $10(8.8)$ & $5(13.1)$ & $5(6.6)$ & 0.04 \\
\hline Secondary & $16(14.0)$ & $9(23.7)$ & $7(9.2)$ & \\
\hline Early latent & $88(77.2)$ & $24(63.2)$ & $64(84.2)$ & \\
\hline
\end{tabular}

D, Treatment

\begin{tabular}{lccc}
\hline Treatment & Overall $(\mathrm{n}=114)$ & Serological cure $(\mathrm{n}=38)$ & Serofast $(\mathrm{n}=76)$ \\
\hline Benzathine penicillin & $92(80.7)$ & $32(84.2)$ & $60(78.9)$ \\
Alternatives & $22(19.3)$ & $6(15.8)$ & $16(21.1)$ \\
\hline
\end{tabular}

E, T0 RPR titer

\begin{tabular}{lccc}
\hline T0 RPR titer & Overall $(\mathrm{n}=114)$ & Serological cure $(\mathrm{n}=38)$ & Serofast $(\mathrm{n}=76)$ \\
\hline$\leq 1: 2$ & $13(11.4)$ & $1(2.6)$ & $12(15.8)$ \\
$1: 4$ & $20(17.5)$ & $5(13.2)$ & $15(19.7)$ \\
$1: 8$ & $26(22.8)$ & $6(15.8)$ & $20(26.3)$ \\
$1: 16$ & $34(29.8)$ & $13(34.2)$ & $21(27.6)$ \\
$1: 32$ & $13(11.4)$ & $8(21.1)$ & $5(6.6)$ \\
$1: 64$ & $6(5.3)$ & $4(10.5)$ & $2(2.6)$ \\
$1: 128$ & $2(1.8)$ & $1(2.6)$ & $1(1.4)$ \\
Geometric mean titer $(95 \% \mathrm{CI})$ & $10(8,12)$ & $16(11,22)$ & $8(6,10)$ \\
\hline
\end{tabular}

F, T1 RPR titer

\begin{tabular}{|c|c|c|c|c|}
\hline T0 RPR titer & Overall $(n=114)$ & Serological cure $(n=38)$ & Serofast $(n=76)$ & $\mathrm{P}$-value \\
\hline$\leq 1: 2$ & $12(10.5)$ & $3(7.9)$ & $9(11.8)$ & $<0.01$ \\
\hline $1: 4$ & $21(18.4)$ & $1(2.6)$ & $20(26.3)$ & \\
\hline $1: 8$ & $37(32.5)$ & $13(34.2)$ & $24(31.6)$ & \\
\hline $1: 16$ & $20(17.5)$ & $7(18.4)$ & $13(17.1)$ & \\
\hline $1: 32$ & $18(15.8)$ & $10(26.3)$ & $8(10.5)$ & \\
\hline $1: 64$ & $6(5.3)$ & $4(10.6)$ & $2(2.7)$ & \\
\hline $1: 128$ & $0(0)$ & $0(0)$ & $0(0)$ & \\
\hline Geometric mean titer $(95 \% \mathrm{CI})$ & $9(8,11)$ & $14(11,19)$ & $8(6,9)$ & $<0.01$ \\
\hline
\end{tabular}

G, T2 RPR titer

\begin{tabular}{lccc}
\hline T0 RPR titer & Overall $(\mathrm{n}=114)$ & Serological cure $(\mathrm{n}=38)$ & Serofast $(\mathrm{n}=76)$ \\
\hline$\leq 1: 2$ & $26(22.8)$ & $15(39.5)$ & $11(14.5)$ \\
$1: 4$ & $32(28.1)$ & $12(31.6)$ & $20(26.3)$
\end{tabular}


Table I. Continued.

\section{G, T2 RPR titer}

\begin{tabular}{lccc}
\hline T0 RPR titer & Overall $(\mathrm{n}=114)$ & Serological cure $(\mathrm{n}=38)$ & Serofast $(\mathrm{n}=76)$ \\
\hline $1: 8$ & $32(28.1)$ & $8(21)$ & $24(31.6)$ \\
$1: 16$ & $17(14.9)$ & $3(7.9)$ & $14(18.4)$ \\
$1: 32$ & $5(4.4)$ & $0(0)$ & $5(6.6)$ \\
$1: 64$ & $2(1.7)$ & $0(0)$ & $2(2.6)$ \\
$1: 128$ & $0(0)$ & $3(0)$ & $0(0)$ \\
Geometric mean titer $(95 \% \mathrm{CI})$ & $6(4,7)$ & $3(2,4)$ & $7(6,9)$ \\
\hline
\end{tabular}

Values are expressed as n (\%) unless indicated otherwise. IQR, interquartile range; RPR, rapid plasma reagin; CI, confidence interval; alternatives, doxycycline or procaine penicillin; T0, before the initial treatment; T1, at 6 months; T2, at 12 months.

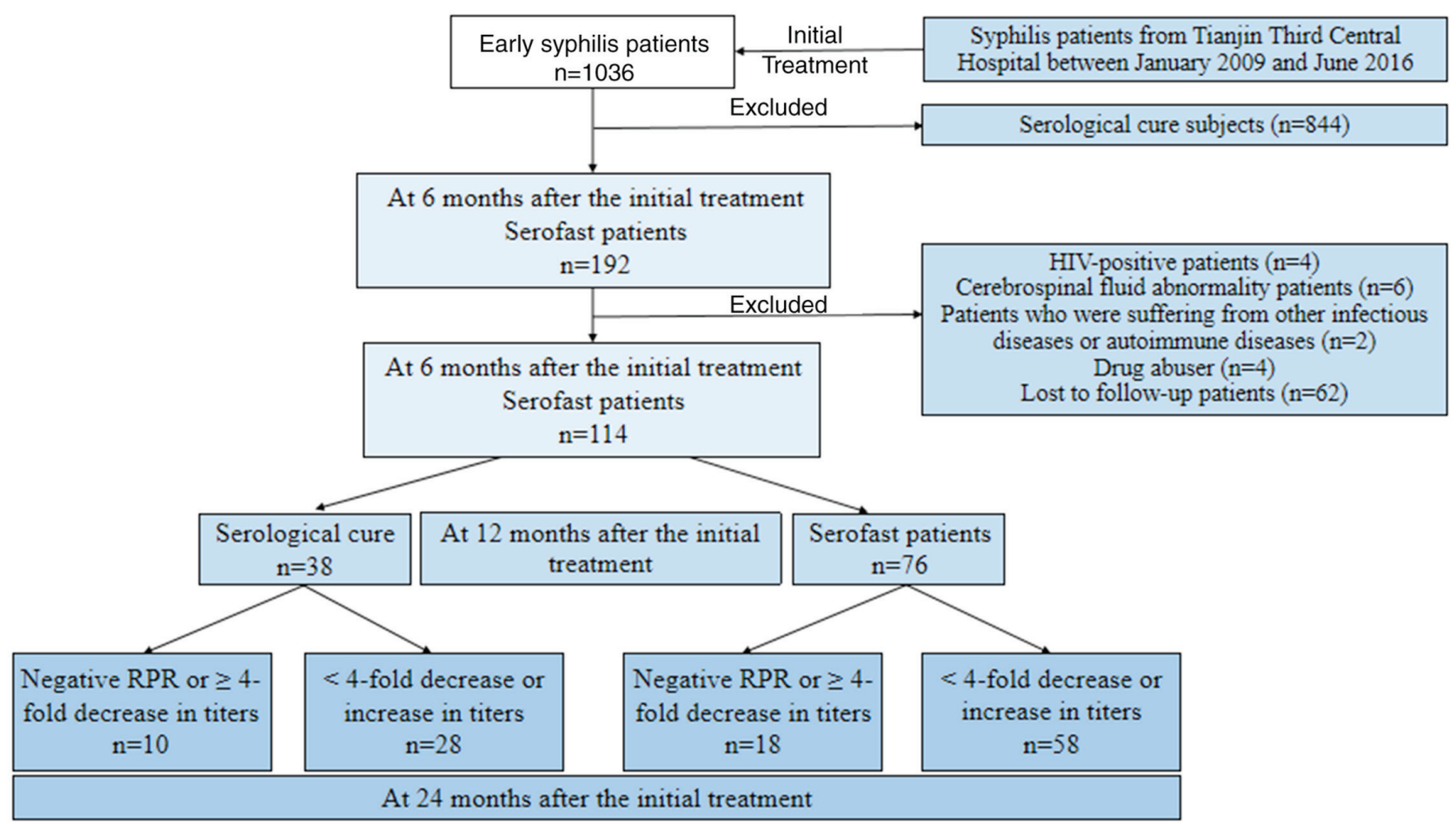

Figure 1. Enrolment and follow-up. RPR, rapid plasma reagin.

patients remained serofast (Table IV). There was no significant difference in sex, age, syphilis type and therapeutic regimen between the two groups $(\mathrm{P}>0.05)$. Of note, a significant difference in T0 (prior to initial treatment) RPR titer, T1 (at 6 months) RPR titer and T3 (at 24 months) RPR titer was seen between serofast patients and serologically cured patients $(\mathrm{P}<0.05)$.

RPR titer at different time-points in serofast patients. The RPR titers in the serofast patients changed at varying time-points. All 114 serofast patients were divided into 7 groups according to their initial RPR titer. Fig. 2 presents the proportion of serofast patients in different RPR titer groups at different time-points. Serological outcomes of serofast patients with early syphilis and different RPR titers at different time-points are also provided in Table $\mathrm{V}$.

\section{Discussion}

After the initial treatment, a total of 114 serofast patients were included in the present retrospective study. Compared to the baseline RPR titers, $33.3 \%$ patients had achieved serological cure after the first retreatment with 2.4 million units of benzathine penicillin weekly for 3 weeks, which was higher than that in the study by Seña et al (27.50\%) (5). There was only 1 dose of benzathine additionally given to serofast patients in the study by Seña et al (5). In the study by Wang et al (9), the serological cure rate was $48.6 \%$. The baseline titers of patients in Wang's study were at a high level, which may be the reason for a higher probability of serological response to therapy (13). Furthermore, the above study had no blank control group. Therefore, a natural decline in RPR titer cannot be completely ruled out in certain patients 
Table II. Characteristics and serological outcomes of serofast patients with early syphilis after the second retreatment.

\section{A, Sex}

\begin{tabular}{|c|c|c|c|c|}
\hline & Overall (n=114) & Serological cure $(n=38)$ & Serofast $(n=76)$ & P-value \\
\hline Female & $49(64.5)$ & $12(66.7)$ & $37(63.8)$ & 0.82 \\
\hline
\end{tabular}

B, Age

\begin{tabular}{|c|c|c|c|c|}
\hline & Overall $(n=114)$ & Serological cure $(n=38)$ & Serofast $(n=76)$ & P-value \\
\hline Median age, years (IQR) & $36(24)$ & $38(20)$ & $35(24)$ & 0.39 \\
\hline
\end{tabular}

C, Syphilis type

\begin{tabular}{|c|c|c|c|c|}
\hline Syphilis type & Overall (n=114) & Serological cure $(n=38)$ & Serofast $(n=76)$ & P-value \\
\hline Primary & $5(6.6)$ & $1(5.6)$ & $4(6.9)$ & $<0.01$ \\
\hline Secondary & $7(9.2)$ & $2(11.2)$ & $5(8.6)$ & \\
\hline Early latent & $64(84.2)$ & $15(83.2)$ & $49(84.5)$ & \\
\hline
\end{tabular}

D, Treatment

\begin{tabular}{lccc}
\hline Treatment & Overall $(\mathrm{n}=114)$ & Serological cure $(\mathrm{n}=38)$ & Serofast $(\mathrm{n}=76)$ \\
\hline Benzathine penicillin & $61(80.3)$ & $15(83.3)$ & $46(79.3)$ \\
Alternatives & $15(19.7)$ & $3(16.7)$ & 0.07 \\
\hline
\end{tabular}

\section{E, T2 RPR titer}

\begin{tabular}{|c|c|c|c|c|}
\hline T2 RPR titer & Overall $(n=114)$ & Serological cure $(n=38)$ & Serofast $(n=76)$ & P-value \\
\hline$\leq 1: 2$ & $11(14.5)$ & $1(5.6)$ & $10(17.2)$ & 0.03 \\
\hline $1: 4$ & $20(26.3)$ & $3(16.2)$ & $17(29.3)$ & \\
\hline $1: 8$ & $24(31.6)$ & $5(27.9)$ & $19(32.8)$ & \\
\hline $1: 16$ & $14(18.4)$ & $5(27.9)$ & $9(15.5)$ & \\
\hline $1: 32$ & $5(6.6)$ & $2(11.2)$ & $3(5.2)$ & \\
\hline $1: 64$ & $2(2.6)$ & $2(11.2)$ & $0(0)$ & \\
\hline $1: 128$ & $0(0)$ & $0(0)$ & $0(0)$ & \\
\hline Geometric mean titer $(95 \% \mathrm{CI})$ & $7(6,9)$ & $11(7,17)$ & $6(5,8)$ & 0.04 \\
\hline
\end{tabular}

\section{F, T3 RPR titer}

\begin{tabular}{|c|c|c|c|c|}
\hline T3 RPR titer & Overall $(n=114)$ & Serological cure $(n=38)$ & Serofast $(n=76)$ & P-value \\
\hline$\leq 1: 2$ & $18(23.7)$ & $7(38.9)$ & $11(18.9)$ & $<0.01$ \\
\hline $1: 4$ & $23(30.3)$ & $7(38.9)$ & $16(27.6)$ & \\
\hline $1: 8$ & $22(29.0)$ & $2(11.1)$ & $20(34.5)$ & \\
\hline $1: 16$ & $11(14.5)$ & $2(11.1)$ & $9(15.5)$ & \\
\hline $1: 32$ & $2(2.5)$ & $0(0)$ & $2(3.5)$ & \\
\hline $1: 64$ & $0(0)$ & $0(0)$ & $0(0)$ & \\
\hline $1: 128$ & $0(0)$ & $0(0)$ & $0(0)$ & \\
\hline Geometric mean titer $(95 \% \mathrm{CI})$ & $5(4,6)$ & $3(2,5)$ & $6(5,7)$ & $<0.01$ \\
\hline
\end{tabular}

Values are expressed as n (\%) unless indicated otherwise. IQR, interquartile range; RPR, rapid plasma reagin; CI, confidence interval; alternatives, doxycycline or procaine penicillin; T2, at 12 months; T3, at 24 months. 
Table III. Serological outcomes in serofast patients with early syphilis after two retreatments.

\begin{tabular}{|c|c|c|c|}
\hline Group & Negative RPR or $\geq 4$-fold decrease in titers & $<4$-fold decrease or increase in titers & Overall \\
\hline First retreatment & $10(26.3)$ & $28(73.7)$ & $38(33.3)$ \\
\hline Second retreatment & $18(23.7)$ & $58(76.3)$ & $76(66.7)$ \\
\hline Overall & $28(24.6)$ & $86(75.4)$ & $114(100)$ \\
\hline
\end{tabular}

$\mathrm{P}$-value $=0.758$. Values are expressed as n $(\%)$ unless indicated otherwise. The 'First retreatment' group, did not receive a second retreatment but were followed up at 24 months to observe the natural change in RPR titer. RPR, rapid plasma regain.

Table IV. Characteristics and serological outcomes of serofast patients with early syphilis after two retreatments.

\begin{tabular}{|c|c|c|c|c|}
\hline \multicolumn{5}{|l|}{ A, Sex } \\
\hline & Overall $(n=114)$ & Serological cure $(n=38)$ & Serofast $(n=76)$ & P-value \\
\hline Female & $69(60.5)$ & $32(57.1)$ & $37(63.8)$ & 0.47 \\
\hline \multicolumn{5}{|l|}{ B, Age } \\
\hline & Overall $(n=114)$ & Serological cure $(n=38)$ & Serofast $(n=76)$ & P-value \\
\hline Median age, years (IQR) & $36(23)$ & $37(22)$ & $35(24)$ & 0.81 \\
\hline \multicolumn{5}{|l|}{ C, Syphilis type } \\
\hline Syphilis type & Overall $(n=114)$ & Serological cure $(n=38)$ & Serofast $(n=76)$ & P-value \\
\hline Primary & $10(8.8)$ & $6(10.7)$ & $4(6.9)$ & 0.15 \\
\hline Secondary & $16(14)$ & $11(19.6)$ & $5(8.6)$ & \\
\hline Early latent & $88(77.2)$ & $39(69.7)$ & $49(84.5)$ & \\
\hline \multicolumn{5}{|l|}{ D, Treatment } \\
\hline Treatment & Overall $(n=114)$ & Serological cure $(n=38)$ & Serofast $(n=76)$ & P-value \\
\hline Benzathine penicillin & $92(80.7)$ & $46(82.1)$ & $46(79.3)$ & 0.89 \\
\hline Alternatives & $22(19.3)$ & $10(17.9)$ & $12(20.7)$ & \\
\hline
\end{tabular}

\section{E, T0 RPR titer}

\begin{tabular}{lccc}
\hline T0 RPR titer & Overall $(\mathrm{n}=114)$ & Serological cure $(\mathrm{n}=38)$ & Serofast $(\mathrm{n}=76)$ \\
\hline$\leq 1: 2$ & $13(11.4)$ & $1(1.8)$ & $12(20.7)$ \\
$1: 4$ & $20(17.5)$ & $7(12.5)$ & $13(22.4)$ \\
$1: 8$ & $26(22.8)$ & $10(17.9)$ & $16(27.6)$ \\
$1: 16$ & $34(29.8)$ & $21(37.5)$ & $13(22.4)$ \\
$1: 32$ & $13(11.4)$ & $9(16.1)$ & 0.01 \\
$1: 64$ & $6(5.3)$ & $6(10.6)$ & $0(0)$ \\
$1: 128$ & $2(1.8)$ & $2(3.6)$ & $7(5,8)$ \\
Geometric mean titer $(95 \% \mathrm{CI})$ & $10(8,12)$ & $16(13,21)$ & $<0.01$ \\
\hline
\end{tabular}

\section{F, T1 RPR titer}

\begin{tabular}{lccr}
\hline T0 RPR titer & Overall $(\mathrm{n}=114)$ & Serological cure $(\mathrm{n}=38)$ & Serofast $(\mathrm{n}=76)$ \\
\hline$\leq 1: 2$ & $12(10.5)$ & $4(7.2)$ & $8(13.8)$ \\
$1: 4$ & $21(18.4)$ & $2(3.6)$ & $19(32.8)$
\end{tabular}


Table IV. Continued.

F, T1 RPR titer

\begin{tabular}{lccr}
\hline T0 RPR titer & Overall $(\mathrm{n}=114)$ & Serological cure $(\mathrm{n}=38)$ & Serofast $(\mathrm{n}=76)$ \\
\hline $1: 8$ & $37(32.5)$ & $20(35.7)$ & $17(29.3)$ \\
$1: 16$ & $20(17.5)$ & $12(21.4)$ & $8(13.8)$ \\
$1: 32$ & $18(15.8)$ & $12(21.4)$ & $6(10.3)$ \\
$1: 64$ & $6(5.3)$ & $6(10.7)$ & $0(0)$ \\
$1: 128$ & $0(0)$ & $0(0)$ & $0(0)$ \\
Geometric mean titer $(95 \% \mathrm{CI})$ & $9(8,11)$ & $14(11,17)$ & $6(5,8)$ \\
\hline
\end{tabular}

G, T2 RPR titer

\begin{tabular}{lccc}
\hline T2 RPR titer & Overall $(\mathrm{n}=114)$ & Serological cure $(\mathrm{n}=38)$ & Serofast $(\mathrm{n}=76)$ \\
\hline$\leq 1: 2$ & $26(22.8)$ & $16(28.6)$ & $10(17.2)$ \\
$1: 4$ & $32(28.1)$ & $15(26.8)$ & $17(29.3)$ \\
$1: 8$ & $32(28.1)$ & $13(23.2)$ & $19(32.8)$ \\
$1: 16$ & $17(14.9)$ & $8(14.2)$ & $9(15.5)$ \\
$1: 32$ & $5(4.4)$ & $2(3.6)$ & $3(5.2)$ \\
$1: 64$ & $2(1.7)$ & $2(3.6)$ & $0(0)$ \\
$1: 128$ & $0(0)$ & $0(0)$ & $0(0)$ \\
Geometric mean titer $(95 \% \mathrm{CI})$ & $6(5,7)$ & $5(4,7)$ & $6(5,8)$ \\
\hline
\end{tabular}

H, T3 RPR titer

\begin{tabular}{|c|c|c|c|c|}
\hline T3 RPR titer & Overall $(n=114)$ & Serological cure $(n=38)$ & Serofast $(n=76)$ & P-value \\
\hline$\leq 1: 2$ & $40(35.1)$ & $29(51.4)$ & $11(9.0)$ & $<0.01$ \\
\hline $1: 4$ & 34 (29.9) & $18(32.4)$ & $16(27.6)$ & \\
\hline $1: 8$ & $26(22.8)$ & $6(10.8)$ & $20(34.5)$ & \\
\hline $1: 16$ & $11(9.6)$ & $2(3.6)$ & $9(15.5)$ & \\
\hline $1: 32$ & $3(2.6)$ & $1(1.8)$ & $2(3.4)$ & \\
\hline $1: 64$ & $0(0)$ & $0(0)$ & $0(0)$ & \\
\hline $1: 128$ & $0(0)$ & $0(0)$ & $0(0)$ & \\
\hline Geometric mean titer $(95 \% \mathrm{CI})$ & $4(3,5)$ & $3(2,4)$ & $6(5,7)$ & $<0.01$ \\
\hline
\end{tabular}

Values are expressed as $\mathrm{n}(\%)$ unless indicated otherwise. IQR, interquartile range; RPR, rapid plasma regain; CI, confidence interval; alternatives, doxycycline or procaine penicillin; T0, before the initial treatment; T1, at 6 months; T2, at 12 months; T3, at 24 months.

that achieved serological cure. The serological response of patients who remained serofast after the first retreatment and received a second retreatment was also evaluated. Patients that achieved serological cure after the first retreatment that received no further retreatment were used as a control for the group with the second retreatment. At 24 months, $23.7 \%$ of patients $(18 / 76)$ had achieved serological cure in the second retreatment group. By contrast, $26.3 \%$ of patients $(10 / 38)$ in the control group exhibited a $\geq 4$-fold decline in RPR titer or negative status. The second retreatment did not significantly improve the serological cure rate in comparison to this control. Similarly, the study by Seña et al demonstrated minimal improvement in serological response among serofast early syphilis patients re-treated with benzathine penicillin, although these patients received only one retreatment (5). In addition, the study by Wang et al suggested that the incremental benefit of re-retreatment in serofast patients with early syphilis was moderate (9).

The present study demonstrated no significant benefit to multiple retreatment of patients with early syphilis and serofast. Not pursuing this avenue of treatment may help to avoid expensive over-treatment of such patients and reduce the financial burden on patients' families and the health system of governments (14). A reduction in retreatment may be beneficial as over-use or abuse of penicillin may lead to drug resistance, causing future damage to patient health. Furthermore, the present results may provide insight into the management of patients with early syphilis with serofast status. It is difficult for 
Table V. Serological outcomes in serofast patients with early syphilis with different RPR titers.

\begin{tabular}{|c|c|c|c|c|c|c|c|c|}
\hline \multirow[b]{2}{*}{$\begin{array}{l}\text { RPR } \\
\text { titer }\end{array}$} & \multicolumn{4}{|c|}{ T2 (at 12 months) } & \multicolumn{4}{|c|}{ T3 (at 24 months) } \\
\hline & Negative & $\begin{array}{l}\geq 4 \text {-fold } \\
\text { decrease }\end{array}$ & $\begin{array}{c}\text { 2-fold } \\
\text { decrease }\end{array}$ & $\begin{array}{l}\text { No change } \\
\text { or increase }\end{array}$ & Negative & $\begin{array}{l}\geq 4 \text {-fold } \\
\text { decrease }\end{array}$ & $\begin{array}{c}\text { 2-fold } \\
\text { decrease }\end{array}$ & $\begin{array}{l}\text { No change } \\
\text { or increase }\end{array}$ \\
\hline$\leq 1: 2$ & 0 & 0 & 2 & 10 & 0 & 0 & 0 & 12 \\
\hline $1: 4$ & 0 & 5 & 6 & 9 & 3 & 2 & 3 & 10 \\
\hline $1: 8$ & 0 & 6 & 8 & 12 & 0 & 5 & 4 & 11 \\
\hline 1:16 & 0 & 14 & 13 & 8 & 0 & 20 & 9 & 5 \\
\hline $1: 32$ & 0 & 8 & 4 & 1 & 1 & 16 & 2 & 2 \\
\hline 1:64 & 0 & 4 & 1 & 1 & 2 & 5 & 0 & 0 \\
\hline $1: 128$ & 0 & 1 & 1 & 0 & 0 & 2 & 0 & 0 \\
\hline
\end{tabular}

RPR, rapid plasma reagin.

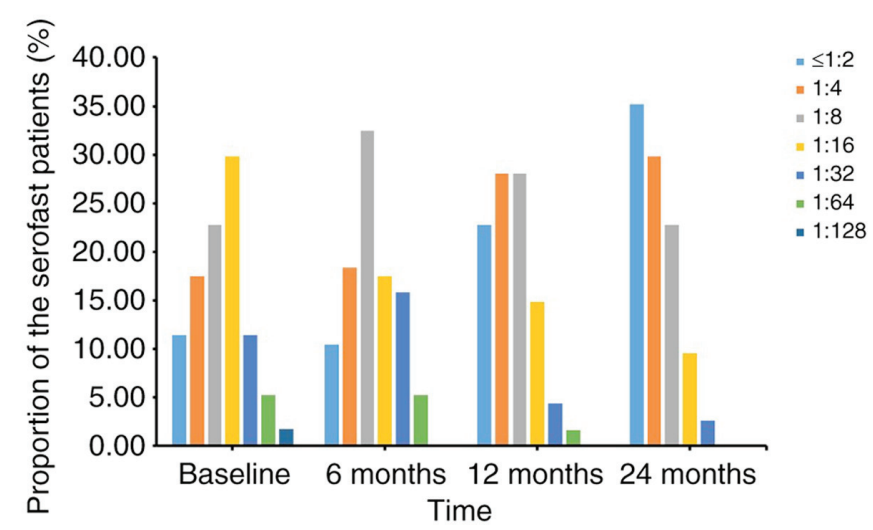

Figure 2. Proportion of serofast patients stratified by their initial rapid plasma reagin titer at different time-points.

clinicians to assess the biological significance of the serofast state (15). Although nontreponemal tests, such as the toluidine red unheated serum test, the rapid plasma reagin serological test and the venereal disease research laboratory slide test, are able to measure IgG and IgM antibodies to TP and potentially to cardiolipin and lipoidal material released from damaged host cells during syphilis infection, other illnesses that produce tissue damage may also lead to positive results (16). In clinical practice, it is a challenge to manage patients with syphilis at the serofast stage using the recommended treatments. Whether there is a persistence of reagin in the circulating blood, persistent spirochetes foci or progressive lesions in patients with syphilis following anti-syphilitic therapy remains unclear (3). The optimal treatment protocol still requires clarification due to insufficient availability of data on the serological response to retreatment and long-term outcomes. In the management of early syphilis at the serofast stage, the variability of the host response to infection should be taken into consideration, and possibly other confounding non-TP inflammatory conditions in the host, including autoimmune diseases, or other infections when stable non-TP antibody titers prevail after treatment (17-19). It is necessary to elucidate the biological basis of serofast and determine whether patients with early syphilis reaching serofast should undergo continued serological monitoring and retreatments in future studies.
To the best of our knowledge, the present study provides the first evaluation of the serological response following a second retreatment of serofast syphilis. However, there are certain limitations. First, the data originated from a retrospectively enrolled cohort, and this retrospective study design is generally considered inferior to a prospective study design. Second, a natural decline in RPR titer cannot be completely ruled out in certain patients with serological cure, as there was no control in the first retreatment for ethical reasons. Third, the different characteristic baselines (the RPR titer and syphilis stage) between the first retreatment group and the second retreatment group may have been a source of bias. The impact of RPR titers and syphilis stage on the curative effect of treatment requires further study. Finally, while the patients with early syphilis in the present cohort study were recruited retrospectively, all of the eligible patients were from a single center and a large number of patients were excluded due to information loss, and the sample size was relatively small.

In conclusion, the present study suggested that patients with early syphilis who reached serofast after the first treatment did not receive any significant benefits after repeated retreatment. In particular, a second retreatment did not significantly improve the patients' serological cure rate. A persistent non-TP serological response after treatment makes it difficult for clinicians to judge the curative effect of treatment. In clinical practice, repeated treatments are controversial. Therefore, in addition to developing a more accurate evaluation method for remedial effects in patients with syphilis, it may be required to fully consider the potential benefits and risks before authorizing repeated retreatments.

\section{Acknowledgements}

Not applicable.

\section{Funding}

No funding was received.

\section{Availability of data and materials}

The datasets used and/or analyzed during the present study are available from the corresponding author on reasonable request. 


\section{Authors' contributions}

YL and SZ designed the study, performed the investigation and data analysis and wrote the manuscript. QB, SZ and JW were responsible for data entry. ZW and JL carried out the RPR test. All of the authors read and approved the manuscript.

\section{Ethics approval and consent to participate}

This study was approved by the Institutional Ethics Committee of Tianjin Third Central Hospital (Tianjin, China). Data were extracted from the medical records of the patients in a manner in which subjects could not be identified; hence, the requirement for informed consent was waived.

\section{Patient consent for publication}

Not applicable.

\section{Competing interests}

The authors declare that they have no competing interests.

\section{References}

1. Cai SN, Long J, Chen C, Wan G and Lun WH: Incidence of asymptomatic neurosyphilis in serofast Chinese syphilis patients. Sci Rep 7: 15456, 2017.

2. Edmondson DG, Hu B and Norris SJ: Long-term in vitro culture of the syphilis spirochete Treponema pallidum subsp. pallidum. MBio 9: e01153-18, 2018.

3. Ren RX, Wang LN, Zheng HY and Li J: No improvement in serological response among serofast latent patients retreated with benzathine penicillin. Int J STD AIDS 27: 58-62, 2016.

4. Kingston M, French P, Higgins S, McQuillan O, Sukthankar A, Stott C, McBrien B, Tipple C, Turner A, Sullivan AK, et al: UK national guidelines on the management of syphilis 2015. Int J STD AIDS 27: 421-446, 2016

5. Seña AC, Wolff M, Behets F, Van Damme K, Martin DH, Leone P, McNeil L and Hook EW: Response to therapy following retreatment of serofast early syphilis patients with benzathine penicillin. Clin Infect Dis 56: 420-422, 2013.

6. Workowski KA and Bolan GA: Sexually transmitted diseases treatment guidelines, 2015. MMWR Recomm Rep 64: 1-137, 2015 .
7. Tuddenham S and Ghanem KG: Emerging trends and persistent challenges in the management of adult syphilis. BMC Infect Dis 15: $351,2015$.

8. SBTS/MOH: National Standard of the People's Republic of China: Diagnostic Criteria and Management of Syphilis (GB 15974-1995). Standards Press of China, Beijing, 1996.

9. Wang ZS, Liu XK and Li J: Serological response to therapy following retreatment of serofast early syphilis patients with benzathine penicillin. J Antimicrob Chemother 73: 1348-1351, 2018.

10. Yang S, Wu J, Ding C, Cui Y, Zhou Y, Li Y, Deng M, Wang C, $\mathrm{Xu} \mathrm{K}$, Ren J, et al: Epidemiological features of and changes in incidence of infectious diseases in China in the first decade after the SARS outbreak: An observational trend study. Lancet Infect Dis 17: 716-725, 2017.

11. Zhang RL, Wang QQ, Zhang JP and Yang LJ: Molecular subtyping of Treponema pallidum and associated factors of serofast status in early syphilis patients: Identified novel genotype and cytokine marker. PLoS One 12: e0175477, 2017.

12. Liang X, Liu T, Yuan C, Wang W and Liang P: The disappearance of femoral head and neck resulting from extensive bone defect caused by secondary syphilis: A case report and literature review. BMC Musculoskelet Disord 19: 251, 2018.

13. Li J, Wang LN and Zheng HY: Predictors of serological cure and serofast state after treatment in HIV-negative patients with early syphilis in China. Sex Transm Infect 89: 69, 2013.

14. Chen ZQ, Zhang GC, Gong XD, Lin C, Gao X, Liang GJ, Yue XL, Chen XS and Cohen MS: Syphilis in China: Results of a national surveillance programme. Lancet 369: 132-138, 2007.

15. Sena AC, Zhang XH, Li T, Zheng HP, Yang B, Yang LG, Salazar JC, Cohen MS, Moody MA, Radolf JD and Tucker JD: A systematic review of syphilis serological treatment outcomes in HIV-infected and HIV-uninfected persons: Rethinking the significance of serological non-responsiveness and the serofast state after therapy. BMC Infect Dis 15: 479, 2015.

16. Macfarlane DE, Hare K and Elias-Jones TF: Evaluation of automated large-scale screening tests for syphilis. J Clin Pathol 29: 317-321, 1976.

17. Larsen SA, Steiner BM and Rudolph AH: Laboratory diagnosis and interpretation of tests for syphilis. Clin Microbiol Rev 8: $1-21,1995$.

18. Schultz DR: Antiphospholipid antibodies: Basic immunology and assays. Semin Arthritis Rheum 26: 724-739, 1997.

19. Augenbraun M, French A, Glesby M, Sanchez-Keeland L, Young M, Greenblatt R and Sharma A: Hepatitis C virus infection and biological false-positive syphilis tests. Sex Transm Infect 86: 97-98, 2010.

This work is licensed under a Creative Commons Attribution-NonCommercial-NoDerivatives 4.0 International (CC BY-NC-ND 4.0) License. 